\title{
La COVID-19, cuando la tragedia se convierte en oportunidad. En busca de una bioética inclusiva
}

\author{
Soledad Arnau Ripollés \\ Universidad Nacional de Educación a Distancia \\ soledadarnauripolles@gmail.com
}

Fecha de recepción: 16-6-2020

Fecha de aceptación: 30-6-2020

\section{Resumen}

En este artículo vamos a reflexionar sobre la situación de las personas con diversidad funcional en tiempos de COVID-19. Esta pandemia actual ha comportado una tragedia sanitaria, social, laboral, educativa y económica. Si partimos de un primer escenario en el que, ya de por sí, dicho grupo social está marginado y sufre discriminación sistemática constantemente, todo ello se agrava ante una situación tan particular como la que ha creado dicha pandemia. Ha resultado necesario establecer criterios de priorización en la atención a pacientes. Así, el derecho universal a la salud ha dejado de ser "tan universal», al mismo tiempo que se puede haber convertido en lo contrario a un derecho. Los protocolos y los criterios de actuación puede que vulneren o violen los derechos humanos de las personas con diversidad funcional o con otras diversidades. Es fundamental que se constituya una nueva línea de investigación-acción desde la diversidad funcional en el campo de la bioética, la cual, denunciada como no neutral, precisa de una reconstrucción. Para que ello sea posible, las personas con diversidad funcional debemos intervenir en el diseño de dichos criterios y protocolos sociosanitarios, aunque solo vamos a poder participar si se nos reconoce nuestra diferencia desde la dignidad inherente e intrínseca. La teoría del reconocimiento de la diversidad va a ser condición indispensable para el desarrollo de una bioética inclusiva. $\mathrm{Y}$ desde este reconocimiento podremos realizar una distribución y una redistribución (mucho mejor) justa y equitativa, además de ética.

Palabras clave: diversidad funcional; teoría del reconocimiento; teoría de la redistribución; justicia distributiva; teoría del reconocimiento de la diversidad; criterios de priorización sanitarios; recursos sanitarios limitados

Abstract. COVID-19, when tragedy turns into opportunity: In search of an inclusive bioethics

In this article we reflect on the situation of people with functional diversity in times of COVID-19. The current pandemic has given rise to a terrible health, social, labor, educational and economic tragedy. The initial scenario of this social group, which was already marginalized and constantly suffered systematic discrimination, has been further aggravated by the specific circumstances caused by the pandemic, where it has become necessary to establish prioritization criteria in patient care. Thus, the universal right to health is no longer "so universal" at a time when it may have become a right for everyone. The protocols and criteria for action may infringe/violate the rights of people with functional diversity, when 
compared to other human diversities. It is essential that a new line of research/action be established regarding functional diversity in the field of bioethics. Bioethics, denounced as non-neutral, needs to be reconstructed. For this to be possible, people with functional diversity must participate in designing these criteria and socio-health protocols. We will only be able to participate if our difference is recognized from our inherent and intrinsic dignity. The theory of the recognition of diversity is going to be an indispensable point in the development of inclusive bioethics. Only when this is recognized, will it be possible to achieve a much fairer and ethical/equitable distribution/redistribution.

Keywords: functional diversity; recognition theory; redistribution theory; distributive justice; diversity recognition theory; health prioritization criteria; limited health resources

\section{Sumario}

1. Introducción $\quad 6$. Tercer escenario: ámbito teórico-

2. Primer escenario: pre-COVID-19 práctico

3. Derecho a la salud 7. A modo de conclusión

4. El modelo biomédico y el capacitismo Referencias bibliográficas

5. Segundo escenario: COVID-19

En una situación de pandemia prevalece el deber de planificar, el principio de justicia distributiva y la maximización del beneficio global. Se debe tener consciencia de la justa asignación de la distribución de recursos sanitarios limitados.

(SEMICYUC, 2020: 5)

\section{Introducción}

De acuerdo con la Convención Internacional sobre los Derechos de las Personas con Discapacidad (CDPD), adoptada por la Asamblea General de las Naciones Unidas el 13 de diciembre de 2006, durante el sexagésimo primer periodo de sesiones de la Asamblea General, por la Resolución 61/106, define a las personas con discapacidad como:

[...] aquellas que tengan deficiencias físicas, mentales, intelectuales o sensoriales a largo plazo que, al interactuar con diversas barreras, puedan impedir su participación plena y efectiva en la sociedad, en igualdad de condiciones con las demás.

Sin embargo, y aunque el término normativo oficial es el de persona con discapacidad, la autora de este trabajo prefiere defender el nuevo concepto de personas con diversidad funcional o bien personas discriminadas por su diversidad 
funcional (Romañach Cabrero y Lobato Galindo, 2007), a fin de utilizar un lenguaje que entiende que es no capacitista, y por tanto no segregador.

Según el Instituto Nacional de Estadística (INE, 2008) ${ }^{1}$, se indica que el $8,5 \%$ de la población total española tiene algún tipo de diversidad funcional (discapacidad) y que a partir de los 45 años hay más mujeres que varones, matiz que se reafirma también por parte del Ministerio de Trabajo y Asuntos Sociales (MTAS, 2006), siguiendo la EDDES-99:

Entre los grupos de gran dependencia o dependencia severa, el 63\% de las personas dependientes son mujeres con discapacidad frente al $37 \%$ de hombres.

\section{Primer escenario: pre-COVID-19}

Según se determina en distintos documentos de Naciones Unidas, el 10\% de la población mundial se encuentra en situación de diversidad funcional por un motivo u otro. Tal y como se expresa en el Informe de la ONU, del Alto Comisionado de Derechos Humanos (OACDH) de Naciones Unidas y la Unión Interparlamentaria (2007: 1), sabemos que las personas con diversidad funcional viven sus vidas en importantes cotas de desigualdad estructural de manera cotidiana:

En todas las regiones del mundo, en cada uno de los países del mundo, las personas con discapacidad viven con frecuencia al margen de la sociedad, privadas de algunas de las experiencias fundamentales de la vida. Tienen escasas esperanzas de asistir a la escuela, obtener empleo, poseer su propio hogar, fundar una familia y criar a sus hijos, disfrutar de la vida social o votar.

Esto nos ayuda a situarnos en un escenario bastante desalentador a nivel internacional. Tal y como explica Devandas-Aguilar (2020: 11):

Tradicionalmente la sociedad ha ignorado o infravalorado la diferencia de la discapacidad y, por tanto, las estructuras sociales no han tenido en cuenta los derechos de las personas con discapacidad.

La categoría analítica de discapacidad conlleva una fuerte discriminación contra las personas que se encuentran ante esta circunstancia. A lo largo del tiempo y en distintos contextos socioculturales, la realidad de la discapacidad es interpretada como enfermedad, como aquello no deseable; aquello defectuoso; aquello por lo que hay que trabajar, en particular por parte de la biotecnociencia, para abolirla en la medida de las posibilidades; aquello a lo que se interrelaciona con la pobreza...

1. Se han realizado tres macroencuestas: la Encuesta sobre discapacidades, deficiencias y minusvalías (EDDM), en 1986; la Encuesta sobre discapacidades, deficiencias y estado de salud (EDDES), en 1999, y la Encuesta de discapacidad, autonomía personal y situaciones de dependencia (EDAD), en 2008. 
Si echamos un vistazo a nivel estatal, vemos que las personas con diversidad funcional estamos en un imaginario colectivo bastante desconcertante. Tal y como comenta el Comité de Bioética de España (2020: 11), existe:

[...] la evidencia de que todas las personas con discapacidad constituyen todavía en nuestro país un grupo social sujeto a una discriminación estructural y sistemática [...].

De aquí que España viese relevante ratificar la CDPD en 2008 y que la población con diversidad funcional lo haya aplaudido. Sin embargo, en nuestro escenario público y político sigue habiendo incongruencias, normativas "sospechosas» que siguen creando una situación desfavorecedora contra este grupo de personas y desarrollando políticas públicas estatales, podemos decir hasta cierto punto, contrarias a la CDPD. A modo de ejemplo:

- Ley 39/2006, de 14 de diciembre, de promoción de la autonomía personal y atención a las personas en situación de dependencia. Tal y como se ha desarrollado a lo largo de estos años, apenas se ha promovido la vida independiente y la autonomía personal como mejor opción (la permanencia residencial e institucional aleja el derecho a vivir en la comunidad, tal y como promueve la CDPD en su artículo 19$)$.

- Ley Orgánica 2/2010, de 3 de marzo, de salud sexual y reproductiva y de la interrupción voluntaria del embarazo. Cabe recordar el artículo 15, "Interrupción por causas médicas». En el apartado $b$ se hace referencia a la posibilidad de abortar cuando no se superen las 22 semanas de gestación y surja el supuesto de graves anomalías en el feto, y en el $c$ se hace hincapié en dos modalidades de supuestos: a las anomalías fetales incompatibles con la vida o a un feto con una enfermedad grave e incurable. Esta normativa sigue defendiendo el aborto eugenésico, con lo cual se sigue considerando que la vida de alguien con diversidad funcional tiene menos valor. Tal y como explica Devandas-Aguilar (2020: 9):

Desde la perspectiva de los derechos de las personas con discapacidad, preocupa que esas prácticas puedan reforzar y validar socialmente el mensaje de que las personas con discapacidad no deberían haber nacido. Los marcos legislativos que amplían los plazos para el aborto legal o, excepcionalmente, permiten el aborto en caso de deficiencia fetal amplifican ese mensaje. [...] las políticas sanitarias y las leyes sobre el aborto que perpetúan estereotipos y estigmas profundamente arraigados contra las personas con discapacidad también socavan la autonomía y libertad de decisión de las mujeres en el ámbito reproductivo.

Siguiendo a nuestro compañero y gran amigo Romañach Cabrero (2012: 6):

[...] la erradicación del aborto eugenésico es el pilar sólido de una nueva construcción de la sociedad, pero que hay muchas más piedras que quitar del camino antes de conseguir el pleno reconocimiento. 
La propuesta de la autora de este artículo, y de acuerdo con la filosofía de vida independiente planteada por el Foro de Vida Independiente y Divertad (FVID), es que se modifiquen los plazos, y si se estima un plazo máximo, que este sea para todos los supuestos, a fin de no promover un imaginario violento contra la condición humana de la diversidad funcional (FVI, 2009).

- Proposición de Ley Orgánica de regulación de la eutanasia (31/01/2020). De acuerdo con el modelo biomédico-rehabilitador-clínico-sociosanitario, quien escribe este artículo tiene diagnosticada de nacimiento la enfermedad de artrogriposis múltiple congénita (AMC) en un $85 \%$. Esto significa que puede interpretarse como una enfermedad grave, en estado crónico e invalidante (tengo una importante movilidad reducida). A ello cabe añadirle una segunda enfermedad, añadida, grave e incurable (cáncer en estadio IV). Esta casuística perfectamente puede encajar adecuadamente con lo que expresa esta proposición de ley en sus artículos 3: «Definiciones», apartados $b$ («Enfermedad grave, crónica e invalidante») y $c$ («Enfermedad grave e incurable»), y 5: «Requisitos para recibir la prestación de ayuda para morir», apartado $d$, donde se nos explica que quien sufra o padezca estas modalidades de enfermedades certificadas por el ámbito médico pueden optar a solicitar la prestación de ayuda para morir).

Desde el activismo de quienes tenemos diversidad funcional, estas dos normativas y esta propuesta de ley pueden ser un ejemplo para hacer evidente que antes de la COVID-19 el escenario para este grupo de personas y sus familias, amistades, parejas, etc. ya no era el mejor de los posibles. La ONU presentó la CDPD en 2006 y España la ratificó en mayo de 2008. Aun así, siguen en vigencia prácticas clínicas como el aborto eugenésico, las esterilizaciones forzosas de muchas mujeres con diversidad intelectual y/o mental o los abortos forzosos como «mejor método anticonceptivo»; prácticas jurídicas como la incapacitación sistemática; prácticas sociales de atención y cuidados como la institucionalización en residencias o centros de día (al respecto, cabe recordar que a partir de determinada edad estos edificios están especialmente feminizados, es decir, ocupados por mujeres con diversidad funcional), o prácticas actitudinales y de comportamientos como las violencias machistas y de género contra las mujeres y personas LGTBQI+ con diversidad funcional en particular.

\section{Derecho a la salud}

No todos podemos tener un acceso igual a los recursos que necesitamos debido a su escasez, en parte inevitable (y en parte no). (Puyol González, 2014: 9)

En el artículo 25, titulado «Salud», de la CDPD (ONU, 2006) se nos explica que las personas con diversidad funcional tenemos: 
[...] derecho a gozar del más alto nivel posible de salud sin discriminación por motivos de discapacidad. Los Estados Partes adoptarán las medidas pertinentes para asegurar el acceso de las personas con discapacidad a servicios de salud $[\ldots]$.

De entre los distintos apartados de este artículo resulta interesante destacar el $d$, en la medida en que hace referencia a que se debe exigir a los profesionales de la salud que presten: «[...] la atención de la misma calidad que las demás personas sobre la base de un consentimiento libre e informado [...] a través de la capacitación y la promulgación de normas éticas para la atención de la salud en los ámbitos público y privado», y el $f$, donde se nos dice que: «Impedirán que se nieguen, de manera discriminatoria, servicios de salud o de atención de la salud o alimentos sólidos o líquidos por motivos de discapacidad».

\section{El modelo biomédico y el capacitismo}

[...] capacitismo: un sistema de valores que considera que determinadas características típicas del cuerpo y la mente son fundamentales para vivir una vida que merezca la pena ser vivida. Atendiendo a estándares estrictos de apariencia, funcionamiento y comportamiento, el pensamiento capacitista considera la experiencia de la discapacidad como una desgracia que conlleva sufrimientos y desventajas y, de forma invariable, resta valor a la vida humana. Como consecuencia de ello, suele inferirse que la calidad de vida de las personas con discapacidad es ínfima, que esas personas no tienen ningún futuro y que nunca se sentirán realizadas ni serán felices. (Devandas-Aguilar, 2020: 3)

El modelo biomédico y el capacitismo se han constituido como dos macrosistemas de poder complementarios que dividen la realidad sociopolítica y cultural en sano / enfermo, normal / anormal, con capacidades / con discapacidades.

Por un lado, desde un discurso de "evidencia científica», el modelo biomédico-clínico-rehabilitador ha marcado a aquellas personas con determinadas diferencias como que estas son deficiencias o patologías. En consecuencia, las personas portadoras de dichas deficiencias o patologías forman parte de ese amplio fenómeno llamado enfermedad, es decir, son concebidas como "personas enfermas». Así, este paradigma hunde sus raíces en el capacitismo y desarrolla políticas identitarias, y todo lo demás configura una extrapolación del ámbito médico-clínico, es decir, construye una serie de roles y patrones comportamentales en función de las enfermedades, deficiencias o patologías y hace que estas determinadas personas «encajen» de acuerdo con su terminología y su definición biomédico-clínica.

Por otro lado, el capacitismo se presenta como el sistema estructural hegemónico de poder que realiza una lectura única de la realidad humana desde el modelo biomédico (y su supuesta objetividad: la evidencia científica), motivo por el que fracciona dicha condición en forma dicotómica. Por tanto, en términos absolutos solo existen "dos clases de personas»: personas "con capacidades» (quienes pueden concebirse como «personas sanas») frente a aquellas 
otras denominadas «con discapacidades» (quienes no terminan de estar sanas). Esta división va mucho más allá de la estricta evidencia científica y otorga privilegios a aquellas personas interpretadas como "con capacidades» (las que comúnmente se autoasignan como «normales») frente a quienes carecen de las mismas y, por tanto, no les queda otra opción, en todo caso, que reclamar sus derechos humanos (cabe recordar que hay una gradación significativa dentro del subgrupo de las personas con discapacidades o con anormalidades que puede influir a la hora de estar en un lado u otro de la balanza).

Tanto el modelo biomédico como el capacitismo construyen lo que se ha denominado «teoría de la tragedia personal», puesto que ambos macrosistemas hacen una interpretación de la tragedia-enfermedad-discapacidad desde una perspectiva individual y anormal. Por este motivo, quien se encuentra en esta circunstancia «sufre» la discapacidad-anormalidad-enfermedad tomando conciencia de que puede ser alguien que jamás consiga curarse o rehabilitarse y por tanto ser una carga familiar, de cuidados y/o socioeconómica.

\section{Segundo escenario: COVID-19}

La crisis de la COVID-19 y el consiguiente estado de alarma en España ha suscitado situaciones que creíamos imposibles hace solo unas pocas semanas, hasta el punto de cuestionar los derechos humanos de los más vulnerables, las personas mayores y las personas con diversidad funcional. (Rodríguez-Picavea Matilla, 2020)

A mediados de marzo de 2020 conocimos el Real Decreto 463/2020, de 14 de marzo, por el que se declaraba el estado de alarma para la gestión de la situación de crisis sanitaria ocasionada por la COVID-19, a causa de lo cual se ha visto afectada la cotidianidad más inmediata propia y ajena. La COVID19 y sus consecuencias han sido devastadoras. Según el Ministerio de Sanidad, Consumo y Bienestar Social (información extraída del documento titulado Actualización n. ${ }^{\circ}$ 131. Enfermedad por SARS-CoV-2 (COVID-19) 09.06.20202), en muy pocos meses han fallecido en nuestro país unas 27.136 personas.

De acuerdo con el Instituto de Salud Carlos III (ISCIII), tal y como explica en su web ${ }^{3}$, el 86\% de personas fallecidas en España tenían más de 70 años (parece que esta pandemia ha sido más dura con las personas mayores y, sobre todo, con las personas mayores con al menos una enfermedad previa).

Así, y ante un escenario tal, donde los servicios hospitalarios de atención a la salud han recibido una cantidad desmesurada de personas por atender, la Sociedad Española de Medicina Intensiva, Crítica y Unidades Coronarias (SEMICYUC) redacta las Recomendaciones éticas para la toma de decisiones en la situación excepcional de crisis por pandemia covid-19 en las unidades de cui-

2. <https://www.mscbs.gob.es/profesionales/saludPublica/ccayes/alertasActual/nCov-China/ documentos/Actualizacion_131_COVID-19.pdf>.

3. <https://cnecovid.isciii.es/covid19/\#grupos-de-poblaci\%C3\%B3n>. 
dados intensivos (2020), a fin de constituir unos criterios de priorización de pacientes (¿quién merece ingresar? y ¿a quiénes asignamos los recursos sanitarios limitados, en este caso, en la unidad de cuidados intensivos?). En estas recomendaciones, se expresa que:

En el caso de desproporción entre la demanda y las posibilidades, es lícito establecer un triaje de ingreso entre los pacientes, basado en el principio de justicia distributiva, evitando seguir el criterio habitual de «el primero en llegar es el primero en recibir asistencia». (SEMICYUC, 2020: 7)

Dentro de las recomendaciones generales se hace hincapié en que hay que valorar al paciente de "forma global» y no la enfermedad de forma aislada (punto 9). En el punto 12 se recuerda el "principio de coste/oportunidad» (hay que ingresar y ofrecer los recursos disponibles a quien tenga más oportunidades de sobrevivir y recuperarse). $Y$ en los puntos 16 y 17 se hace referencia a que hay que proporcionar los recursos sanitarios limitados a quienes vayan a «vivir más años con calidad» (priorizar la mayor esperanza de vida con calidad) y a aquellas personas mayores con "[...] supervivencia libre de discapacidad [...]», respectivamente (SEMICYUC, 2020: 13). Y, dentro de las recomendaciones específicas, también expresa la denegación de la ventilación mecánica invasiva para aquellas personas con deterioro cognitivo (punto 4).

Ante este tipo de manifestaciones públicas, y si ya de por sí el escenario habitual es bastante penoso o, mejor dicho, poco ético, donde los derechos humanos de las personas con diversidad funcional se ven continuamente vulnerados y violados, ni qué decir tiene que pasar a "otro escenario» en el que existe abiertamente una clara crisis de salud pública, materializada en una crisis sociosanitaria, política y económica, puede resultar fatal. En este nuevo panorama, las personas con diversidad funcional o con otras diversidades, si ya venimos de esa situación insostenible de vulnerabilidad como constante, en el momento actual todo se complejiza mucho más, y siempre suele ser para peor (Rodríguez-Picavea Matilla, 2020; SEMICYUC, 2020). El Comité de Bioética de España (2020: 9) entiende que la postura de la SEMICYUC no es compatible con la CDPD.

A todo esto cabe añadir que las medidas de protección y prevención de contagios son complejas para algunas personas a la hora de ejecutarse. Se nos ha pedido y exigido que nos confinemos. Ha habido determinado sector poblacional que ha protestado por esta medida preventiva (por ejemplo: el movimiento en línea Stop Confinamiento España ${ }^{4}$ ). Sin embargo, nadie ha tenido ni tiene presente que muchas personas con diversidad funcional, a lo largo de sus (nuestros) ciclos vitales, hemos sufrido, y sufrimos, un confina-

4. I. Sierra (2020), «\#StopConfinamientoEspaña: El movimiento nacido en internet para terminar con el aislamiento "en vano" ", Magnet (27 de abril). Recuperado de <https:// magnet.xataka.com/en-diez-minutos/stopconfinamientoespana-que-hay-detras-movimientoprotesta-restricciones $>$. 
miento sociohistórico y político, muchas veces por falta de accesibilidad $5 / \mathrm{o}$ por falta de políticas públicas de cuidados adecuadas para que las personas que lo necesitamos dispongamos de personal de apoyo (asistentes personales ${ }^{6}$, intérpretes de lengua de signos, guías intérpretes, mediadores, etc.), personal e individualizadamente y durante una cantidad de horas necesaria.

Así, también ha sido importante pedir que toda la información sobre esta pandemia se realizase en formato de lectura fácil y que algunas personas con diversidad intelectual o mental tenían necesidad de salir a la calle aunque hubiese confinamiento y estado de alarma; que las mascarillas debieran disenarse de tal modo que fuesen accesibles para que quienes leen los labios tuvieran acceso a ello, o que la distancia de seguridad entre unos individuos y otros debiera tener en cuenta a las personas ciegas o con baja visión.

\section{Tercer escenario: ámbito teórico-práctico}

\subsection{La no neutralidad de la bioética}

Históricamente, la bioética y la discapacidad han mantenido una relación estrecha pero conflictiva. [...]. Gran parte de la labor realizada en el campo de la bioética hasta la fecha se ha basado en un entendimiento insuficiente o inexacto de la diversidad y la complejidad de la discapacidad y de su naturaleza intrínsecamente vinculada a la sociedad. (Devandas-Aguilar, 2020: 5-6)

Tal y como se ha expresado en distintos espacios, la bioética no es neutral. A modo de ejemplo, y en lo que se refiere a la ausencia del feminismo y a las cuestiones de género en la disciplina bioética, Marsico (2003: 25) comenta lo siguiente:

[...] la bioética no ha prestado suficiente atención al feminismo, como tampoco a las cuestiones de género. Es posible que la causa de todo esto no resida sólo en los factores de orden social —la minoría de mujeres respecto a los hombres en el ámbito académico, médico y cultural, en general-, sino, sobre todo, en la estructura profunda y en la evolución misma de la bioética.

Por parte de las personas con diversidad funcional también se denuncia esa no neutralidad de la bioética en cuanto que se interpreta que dicha disciplina se fundamenta en el modelo biomédico-rehabilitador-clínico-sociosanitario y el sistema capacitista. Se trata de crear, por tanto, un nuevo discurso teóricopráctico fundamentado en los derechos humanos de este grupo de personas.

5. «Encerrados en casa: Casi 100.000 españoles no salen nunca por problemas de accesibilidad», El Periódico (12 de junio de 2019). Recuperado de <https://www.elperiodico.com/ es/sociedad/20190612/100000-espanoles-nunca-salir-casa-falta-accesibilidad-7500793>.

6. V. Valero Sanchis (2009), "Los asistentes personales», Foro de Vida Independiente y Divertad: Comunidad de reflexión filosófica y de lucha por los derechos de las personas con diversidad funcional. Recuperado de <http://forovidaindependiente.org/los-asistentes-personales/>. 
Tal y como expresa Devandas-Aguilar (2020: 7), tanto la ONU como su organismo la UNESCO necesitan implementar la CDPD:

Si bien la bioética se ha comprometido a promover el respeto de la dignidad humana y proteger los derechos humanos (Declaración Universal sobre Bioética y Derechos Humanos, art. 2), todavía no ha incorporado los derechos de las personas con discapacidad, consagrados en la Convención sobre los Derechos de las Personas con Discapacidad.

En consecuencia, la interesante propuesta de creación de nuevas líneas bioéticas, tales como bioética de/desde la diversidad funcional (Arnau Ripollés, 2011), bioética que nace al otro lado del espejo (Romañach Cabrero, 2009), bioética de la discapacidad o bioética consciente de la discapacidad (Devandas-Aguilar, 2020), hace que se constituyan en discursos bioéticos muy necesarios.

En lo que concierne a la voz bioética deldesde la diversidad funcional, cabe decir que la Disabled People's International ${ }^{7}$ (DPI) (Organización Mundial de Personas con Discapacidad), en especial la organización de la región de Europa, ha jugado un papel muy importante dentro de la disciplina de bioética "vista» desde la diversidad funcional. Así, de acuerdo con el Plan de Acción 1999-2000, de DPI Europa, adoptado en Siracusa (Italia), una de las áreas prioritarias era: «[...] influir en la Unión Europea, el Consejo de Europa y los gobiernos nacionales en su forma de pensar sobre asuntos bioéticos [...]» y «[...] educar a las personas con discapacidad de Europa y del resto del mundo sobre..., bioética».

A principios del 2000 se creó un grupo de trabajo formado por miembros de la DPI Europa de Francia, Italia, Portugal, España ${ }^{8}$ y el Reino Unido para debatir estos asuntos desde la perspectiva de la diversidad funcional y desarrollar una declaración de principios. En ese mismo año dicha entidad organizó la I Conferencia Internacional sobre Bioética y Derechos Humanos, en Solihull (Reino Unido). De este primer encuentro nace la Declaración de Solihull: «El derecho a vivir y a ser diferentes».

\subsection{La justicia distributiva en sanidad}

La bioética, entendida como ética aplicada, hace uso de distintas disciplinas para abordar temas muy complejos que giran en torno a la vida y a la propia muerte. Esta disciplina, que ya está a punto de cumplir 50 años, va configurando su complejidad conforme se van desarrollando los avances biotecno-

7. <http://www.dpi.org/>.

8. En el caso de España, participó el investigador en bioética y diversidad funcional, Javier Romañach Cabrero, en calidad de representante de la Confederación Española de Personas con Discapacidad Física y Orgánica (COCEMFE). Esta entidad es miembro de DPI Europa y suscribió dicho documento.

9. <http://www.diversocracia.org/docs/Bioetica_DPI_solihull_es.pdf $>\mathrm{y}<\mathrm{http} / / \mathrm{dpi}$.org/langsp/resources/topics_detail?page=895>. 
científicos. No es nada sencillo tomar decisiones que cambien el devenir existencial de los individuos y de las colectividades.

Si inicialmente la bioética ha tenido su razón de ser en la creación de puentes entre las ciencias y las humanidades, cierto es que lo ha hecho desde una perspectiva concreta: desde el mundo occidental y con personas con determinadas características (varones, blancos, del norte, judeocristianos, de mediana edad, sin diversidad funcional ni otras diversidades, de clase media, etc.), con lo cual sus discursos y sus prácticas han estado condicionadas.

Tal y como ya se ha dicho, en el caso que nos ocupa, las personas con diversidad funcional mantenemos una relación muy estrecha con la bioética en particular, porque la que se ha desarrollado ha sido principalmente la bioética clínica o la de la investigación. Sin embargo, ha sido sobre todo la clínica la que «ha tratado la discapacidad» a lo largo del tiempo.

Aun así, ha sido la ética principalista la que ha gobernado la disciplina bioética. Han sido los principios éticos básicos (beneficencia, no maleficencia, autonomía y justicia) los que han formado el horizonte normativo que la ha guiado.

Tal y como expresa Ferrer Lues (2003: 117): «La bioética ha mostrado especial énfasis en el principio de autonomía y de los derechos individuales». Si en nuestro contexto occidental el principio de autonomía ha sido el de mayor relevancia dentro de la ética clínica, puesto que vivimos en una sociedad en la que ha predominado la idea del individualismo buscando la horizontalidad entre médico y paciente a través del consentimiento libre e informado, cierto es que en el plano de la ética de la investigación surgen serias dudas (no es lugar para extendernos en ello, pero cabe decir que no todas las personas con diversidad funcional estamos de acuerdo en que se realicen investigaciones con individuos diversos que no pueden representarse a sí mismos).

Sin embargo, en estos últimos tiempos estamos percibiendo un nuevo cambio bioético. El principio de justicia se está extendiendo. Si recordamos las palabras del principio de este artículo, expresadas por la SEMICYUC, porque estamos ante una pandemia «[...] prevalece el deber de planificar, el principio de justicia distributiva y la maximización del beneficio global», todo ello, en definitiva, para "[...] tener consciencia de la justa asignación de la distribución de recursos sanitarios limitados».

Así es, los recursos sanitarios son limitados y ante una crisis de salud pública como la que se acaba de padecer ha habido un claro desajuste entre la demanda de servicios de atención sanitaria, desmesurada, y los medios limitados que pueden ofrecerse (es decir, no ha habido suficientes recursos para atender a todas las personas que tenían necesidad de ser atendidas).

En consecuencia, parece lógico y de sentido común que si habitualmente se establecen criterios de priorización en la asignación de los recursos sanitarios limitados, en momentos como este, ante un desequilibrio tal, se elaboren más criterios. Tal y como comenta el Comité de Bioética de España (2020: 4), dicha priorización es algo que se ha asumido a lo largo del tiempo: 
[...] la priorización en la asignación de recursos sanitarios no es una novedad ni una consecuencia de la pandemia, sino algo inherente a cualquier sistema de salud (véanse, por ejemplo, los triajes en Urgencias, las listas de espera o las decisiones de incluir o no en la cartera de servicios determinados medicamentos o prestaciones sanitarias).

En este contexto hemos visto que, a consecuencia de la pandemia provocada por la COVID-19, el criterio de la edad ha sido puesto en práctica y las personas mayores ${ }^{10}$ han quedado hasta cierto punto excluidas en ese proceso de priorización. Así también la situación de discapacidad ha sido un elemento que tener en cuenta para dicha exclusión. Ahora bien, cabe explicar que apenas se conocen cifras exactas de personas con discapacidad ${ }^{11}$ que hayan fallecido estando en residencias.

Desde la perspectiva de los individuos con diversidad funcional este tipo de exclusiones son interpretadas como una vulneración o una violación de nuestros derechos humanos. España ha ratificado la CDPD, motivo por el cual, tal y como muy acertadamente expresa Devandas-Aguilar (2020: 12), las personas con diversidad funcional hemos adquirido, a través del documento de Naciones Unidas, la «dignidad inherente»:

$\mathrm{La}$ «dignidad inherente» se refiere al valor que tienen todas las personas. El reconocimiento de la dignidad inherente es un recordatorio poderoso de que las personas con discapacidad, por el mero hecho de ser personas, tienen el mismo valor y se merecen el mismo respeto que las demás.

Por tanto, la dignidad inherente o intrínseca es algo que nos pertenece y que sociopolítica y culturalmente, podríamos afirmar, se nos había arrebatado. En este arrebatamiento, la bioética se ha construido al margen de nuestra condición de dignidad intrínseca, de ahí que se haya denunciado su no neutralidad en sus discursos y sus prácticas. Por ello mismo, la pregunta fundamental sería la siguiente: “¿Cómo se puede distribuir ese bien común denominado "recursos sanitarios" sin desproteger los derechos humanos de las personas que se encuentran en situación de mayor vulnerabilidad, como somos las que presentamos diversidad funcional o las de mayor edad?». Posiblemente, una buena respuesta se encuentre en el hecho de cambiar la justicia distributiva por, mejor, una justicia redistributiva. En palabras de Romañach Cabrero (2012: 3):

[...] la premisa clave en las políticas de redistribución está en quién ha sido reconocido como igual con carácter previo. [...] entiendo que el reconocimiento previo es el que determina los criterios a seguir en la redistribución.

10. «El abandono de personas mayores en las residencias durante la pandemia del coronavirus no puede permitirse», Noticias ONU (27 de marzo de 2020). Recuperado de <https://news. un.org/es/story/2020/03/1471932>.

11. " 29 fallecidos por Covid-19 en residencias para personas con discapacidad de la Comunidad de Madrid», Servimedia (21 de abril de 2020). Recuperado de <https://www.servime dia.es/noticias/1245755>. 
La justicia distributiva existe en nuestro país, con lo cual ya tenemos un punto de partida. Ahora bien, ¿es ética o justa la justicia distributiva con las personas más vulnerables? La respuesta por parte de las que mostramos diversidad funcional y que nos dedicamos al mundo de la bioética es rotunda: «iNo! La justicia distributiva no siempre es justa o ética, en particular cuando se trata de aplicarla a personas que se encuentran en situación de especial vulnerabilidad, como las que presentan diversidad funcional o las que son de mayor edad".

En consecuencia, cabe preguntarse: «La justicia distributiva, ¿parte de la teoría del reconocimiento de las personas con diversidad funcional?». Puede que no. El ejemplo de los criterios de priorización presentados por la sociedad científica SEMICYUC apuntan a que la respuesta sea un no.

En la actualidad, gracias a Guibet Lafaye y Romañach Cabrero (2010: 1), estamos conociendo un primer esbozo de lo que son las «teorías éticas (de reconocimiento recíproco) de la diversidad». Estos autores expresan lo siguiente:

Las éticas de la diversidad proporcionan fundamentos sólidos para defender la justicia y la igualdad de todas las diversidades humanas y son una alternativa a otros enfoques que amplían la comunidad moral, excluyendo a algunos seres humanos, como hace la ética utilitarista de Peter Singer.

Estas «éticas de la diversidad» $\mathrm{o}$ "éticas del reconocimiento de la diversidad» se desarrollan principalmente a partir de las teorías éticas modernas sobre el reconocimiento y la redistribución, cuyos máximos representantes son Axel Honneth y Nancy Fraser (Fraser y Honneth, 2006), y del paradigma de la diversidad (Palacios Rizzo y Romañach Cabrero, 2006), que no podemos tratar aquí por falta de tiempo.

Las personas con diversidad funcional, o con otras diversidades humanas, que estemos estigmatizadas sociocultural y políticamente necesitamos que se nos reconozca nuestra dignidad inherente e intrínseca y, a partir de ahí, que se construyan discursos y prácticas clínico-sanitarias respetuosas con la diferencia humana ${ }^{12}$.

Es por este motivo que resulta imprescindible la propuesta de Arnau Ripollés (2011): los comités de bioética estatales, incluyendo el de nuestro país, así como los comités de ética asistencial hospitalarios, necesitan la presencia y la participación de las personas con diversidad funcional. Es inviable que nos sigamos manteniendo al margen, cuando esta disciplina nos toca tan de cerca.

Asimismo, Devandas-Aguilar (2020: 20) realiza la siguiente recomendación en el apartado $g$ ):

Colaborar y consultar activamente con las personas con discapacidad y las organizaciones que las representan en todos los procesos de adopción de decisiones relacionados con la práctica médica y científica que les concierna, lo cual incluye las reformas legislativas, la elaboración de políticas y la realización

12. "Toda persona tiene derecho a cuidados médicos si se infecta con el coronavirus: es un derecho humano", Noticias ONU (26 de marzo de 2020). Recuperado de <https://news. un.org/es/story/2020/03/1471802>. 
de estudios. Los comités nacionales de bioética deben consultar e incluir a las personas con discapacidad en su labor.

\section{A modo de conclusión}

Se puede recordar que sociopolítica y culturalmente necesitamos «rehabilitar» a las personas con diversidad funcional, basándonos en el enfoque de los derechos humanos y devolviéndoles la dignidad inherente e intrínseca que la historia de la evidencia científica biomédico-clínico-sociosanitaria les (nos) ha arrebatado. Esta devolución va a significar una verdadera revolución en todos los ámbitos, incluyendo a la disciplina bioética.

El mundo sanitario precisa de protocolos de actuación a fin de que la toma de decisiones sea lo más rápida y sencilla posible, sobre todo en momentos críticos como pueden ser los provocados por la pandemia actual.

Esta circunstancia «anómala» ha puesto una vez más sobre la mesa la desigualdad estructural que gira en torno a la población con diversidad funcional. Si en momentos de tranquilidad y durante los cuales supuestamente el Estado de bienestar funcionaba medianamente ya había discriminación sistemática contra este grupo social por parte de las instituciones sanitarias y clínicas, ni qué decir tiene que en estos otros momentos, en este otro escenario, la situación es peor.

Se entiende que hay que priorizar, que los recursos son limitados, que los recursos limitados deben distribuirse (o, mejor dicho, «redistribuirse») y que lleguen al máximo posible de pacientes (de ahí la maximización global expresada al inicio), pero no a costa de vulnerar los derechos humanos de quienes manifiestan más fragilidad.

Si queremos una justicia distributiva justa y ética va a ser necesaria la plena participación de las personas con diversidad funcional a la hora de establecer los criterios justos de distribución sanitaria. No podemos continuar siendo exclusivamente un grupo social perceptor de lo que se nos quiera o se nos pueda ofrecer. Más bien, a la hora de realizar la repartición o la redistribución debemos formar parte de esos espacios de poder, en este caso para empoderar, y que los criterios que se determinen sean consensuados por todas las partes implicadas (recordemos que las personas con diversidad funcional estamos directamente implicadas en los avances biomédicos y tecnocientíficos, así como en el propio desarrollo del campo de la bioética).

Por ello mismo, la bioética, disciplina no neutral, necesita incorporar la perspectiva y la voz de las personas con diversidad funcional. Se necesita una bioética «desde» la diversidad funcional que aporte amplitud a la bioética y la haga inclusiva. 


\section{Referencias bibliográficas}

Arnau Ripollés, M.S. (2011). «Comités de Bioética: Nuevos desafíos para la inclusión de la Diversidad Funcional». Medicina y Humanidades: Revista de Medicina, Bioética y Filosofia, 1-2, 59-75.

Comité de Bioética de España (2020). Informe del Comité de Bioética de España sobre los aspectos bioéticos de la priorización de recursos sanitarios en el contexto de la crisis del coronavirus. Madrid: Comité de Bioética de España (CBE). Recuperado de <http://assets.comitedebioetica.es/files/ documentacion/Informe\%20CBE-\%20Priorizacion $\% 20 \mathrm{de} \% 20$ recursos $\% 20$ sanitarios-coronavirus\%20CBE.pdf>.

Devandas-Aguilar, C. (2020). Informe de la Relatora Especial sobre los derechos de las personas con discapacidad (Tema: los efectos del capacitismo en la práctica médica y científica). Ginebra: Oficina del Alto Comisionado para los Derechos Humanos de Naciones Unidas. Recuperado de <https://docu ments-dds-ny.un.org/doc/UNDOC/GEN/G19/346/57/PDF/G1934657. pdf?OpenElement>.

Ferrer Lues, M. (2003). «Equidad y justicia en salud. Implicaciones para la bioética». Acta Bioethica, 9 (1), 113-126. Recuperado de <https://scielo. conicyt.cl/pdf/abioeth/v9n1/art11.pdf>.

Fraser, N. y Honneth, A. (2006). ¿Redistribución o reconocimiento? Madrid: Morata.

FVI (2009). Comentarios al Anteproyecto de ley orgánica de salud sexual y reproductiva y de la interrupción voluntaria del embarazo: Postura del Foro de Vida Independiente. Recuperado de <http://forovidaindependiente.org/wpcontent/uploads/Comentarios_anteproyecto_aborto_FVI_08_2009.doc>.

FVID (2020). «Por la dignidad y contra la indefensión de las personas en situación de dependencia en la crisis del COVID-19». Foro de Vida Independiente y Divertad (FVID): Comunidad de reflexión filosófica y de lucha por los derechos de las personas con diversidad funcional. Recuperado de $<$ http://forovidaindependiente.org/por-la-dignidad-y-contra-la-indefen sion-de-las-personas-en-situacion-de-dependencia-en-la-crisis-delcovid-19/>.

Guibet-Lafaye, C. y Romañach Cabrero, J. (2010). «Diversity Ethics: An alternative to Peter Singer's ethics». Dilemata: Revista Internacional de Éticas Aplicadas, año 2, núm. 3 (mayo). Recuperado de <http://www.dilemata. net/ revista/index.php/dilemata/article/view/37>.

INE (2008). Encuesta sobre discapacidades, autonomía personal y situaciones de dependencia. Madrid: Instituto Nacional de Estadística. Recuperado de <http:// www.ine.es/dyngs/INEbase/es/operacion.htm?c=Estadistica_ C\&cid $=1254736176782 \&$ menu $=$ resultados\&idp $=1254735573175>$.

Marsico, G. (2003). Bioética: Voces de mujeres. Madrid: Narcea.

MTAS (2006). Plan de acción para las mujeres con discapacidad 2007. Madrid: Dirección General de Coordinación de Políticas Sectoriales sobre la Discapacidad. Secretaría de Estado de Servicios Sociales, Familias y Discapaci- 
dad. Ministerio de Trabajo y Asuntos Sociales. Recuperado de <https://sid. usal.es/idocs/F8/FDO18244/pamcd2007.pdf>.

ONU (2006). Convención Internacional sobre los Derechos de las Personas con Discapacidad. Adoptada por la Asamblea General de las Naciones Unidas el 13 de diciembre de 2006, durante el sexagésimo primer periodo de sesiones de la Asamblea General, por la Resolución 61/106. Recuperado de <https://www.un.org/esa/socdev/enable/documents/tccconvs.pdf>.

Palacios Rizzo, A. y Romañach Cabrero, J. (2006). El modelo de la diversidad: La Bioética y los Derechos Humanos como herramientas para alcanzar la plena dignidad en la diversidad funcional. A Coruña: Ediciones DiversitasAlES. Recuperado de <https://e-archivo.uc3m.es/handle/10016/9899>.

Puyol González, Á. (2014). "Justicia distributiva en el sistema de salud: El racionamiento de los recursos en tiempo de crisis». Derecho y Salud, 24 (número extra 1), 6-13. [Ejemplar dedicado al XXIII Congreso Derecho y Salud. Sistema de salud: novedades y desafíos.] Recuperado de <https:// dialnet.unirioja.es/descarga/articulo/5077529.pdf>.

Rodríguez-Picavea Matilla, A. (2020). «El covid 19 arrasa con todo, hasta con los derechos humanos». Derechos Humanos, ;Ya!: Los derechos y la dignidad de las personas con diversidad funcional. Recuperado de <http://dere choshumanosya.org/el-covid-19-arrasa-con-todo-hasta-con-los-derechoshumanos/>.

Romañach Cabrero, J. (2009). Bioética al otro lado del espejo. La visión de las personas con diversidad funcional y el respeto a los Derechos Humanos. A Coruña: Ediciones Diversitas-AlES.

- (2012). Al otro lado del espejo: ¿Reconocimiento como redistribución? Reflexiones éticas y politicas alrededor de los recortes sociales y el aborto eugenésico en España. Recuperado de <http://www.diversocracia.org/docs/aolde_recono cimiento.doc>.

Romañach Cabrero, J. y Lobato Galindo, M. (2007). «Diversidad funcional: Nuevo término para la lucha por la dignidad de la diversidad del ser humano». En: Áluvarez Pousa, L. et al. (coord.). Comunicación y discapacidades: Actas del Foro Internacional. Galicia: Observatorio Gallego de Medios. Colegio Profesional de Periodistas de Galicia, 321-330. Recuperado de <http://dialnet.unirioja.es/servlet/articulo?codigo=2393402>. SEMICYUC (2020). Recomendaciones éticas para la toma de decisiones en la situación excepcional de crisis por pandemia covid-19 en las unidades de cuidados intensivos. Madrid: Sociedad Española de Medicina Intensiva, Crítica y Unidades Coronarias (SEMICYUC). Recuperado de <https://semicyuc. org/wp-content/uploads/2020/03/\%C3\%89tica_SEMICYUC-COVID19.pdf>. 
Soledad Arnau Ripollés es una mujer con diversidad funcional física de nacimiento. Natural de Nules y afincada en Madrid hace más de 18 años, es doctora en Filosofía (UNED), agente de igualdad (UJI-FIIO), bioeticista (UNED, URL-IBB) y sexóloga (UNED, URJC-Fund. Sexpol). Másteres y cursos de especialización en Discapacidad/diversidad funcional; Feminismos, igualdad de géneros, prevención de violencias machistas, nuevas masculinidades; Sexología, sexualidades no hegemónicas, sexualidades y géneros no binarios, educación sexual inclusiva; Filosofía para la paz, educación para la ciudadanía, derechos humanos, educación inclusiva; Bioética, bioética desde la diversidad funcional. Activista feminista y del Movimiento de Vida Independiente en España. Becaria investigadora por parte de distintas instituciones públicas y privadas. Cofundadora de la Oficina de Vida Independiente de la Comunidad de Madrid y primera coordinadora de la misma. Investigadora universitaria y académica en proyectos nacionales y latinoamericanos. Conferenciante y articulista habitual. Ha sido coprotagonista de audiovisuales y directora y presentadora del programa jAcuéstate conmigo!, de radio Yaloveras. Para más información: <www. soledadarnau.com>.

Soledad Arnau Ripollés is a woman with physical functional diversity from birth. Born in Nules and living in Madrid for more than 18 years, she has a PhD in Philosophy (UNED) and is an equality agent (UJI-FIIO), bioethicist (UNED, URL-IBB) and sexologist (UNED, URJCFund. Sexpol). She has completed master degrees and specialization courses in disability/functional diversity; feminisms, gender equality, prevention of sexist violence and new masculinities; sexology, non-hegemonic sexualities, non-binary sexualities and genders and inclusive sexual education; philosophy for peace, education for citizenship, human rights and inclusive education; and bioethics, bioethics from functional diversity. She is also a research fellow at various public and private institutions and university and academic researcher in national and Latin American projects. She is a feminist activist and member of the Independent Life Movement in Spain as well as the co-founder and first co-coordinator of the Independent Living Office of the Community of Madrid. A lecturer and regular columnist, she has co-starred in audiovisual productions and directed and presented the program ;Acuéstate conmigo! on radio Yaloveras. For more information: <www.soledadarnau.com>. 